\title{
Dielectric-Recovery Characteristics of Power Arcs in Large Air Gaps
}

\author{
G. D. McCANN \\ FELLOW AIEE
}

\author{
J. E. CONNER \\ NONMEMBER AIEE \\ H. M. ELLIS \\ STUDENT MEMBER AIEE
}

Synopsis: A satisfactory test technique has been developed for studying the rates of dielectric recovery of large air gaps and other types of power-system insulation. This permits the accurate control of the fault conditions so that all practical types of fault currents can be studied. These are (1) very high magnitude short-duration surges typical of lightning currents; (2) currents of power-system frequencies; and (3) intermediate duration currents such as those which might result from high-frequency current zeros produced by natural system oscillations.

Results are presented showing the rate of dielectric recovery of $3-, 6-$, and 11-inch standard rod gaps for power frequency fault currents up to 700 amperes. Electrode cooling effects were found important at 3-inch gap spacings but not at six inches or above. The 11-inch gap data are proportionately higher than the 6 -inch data, indicating that the results can be extrapolated. The data show that for arcs of a few cycles actual duration has little effect on rate of recovery. A range of current magnitudes from 50 to 700 amperes causes only about a 2-to-1 variation in rate of recovery. For the normal ratios of transmission-line insulation level to operating voltage (about four to one) minimum delay times of from 0.025 second for 100 -ampere faults to 0.05 second for 700 ampere faults are required before the recovery voltage reaches the magnitude of the normal applied voltage. Time intervals of 0.05 to 0.08 second are required before the voltage strength has built up to twice this level or one-half the initial gap strength. This is contrasted with a corresponding time interval of only 2- to 3,000-microseconds for very short duration lightning-surge currents.

High-speed camera studies of the arcs show that for power-frequency faults of onehalf to one-cycle duration, visible luminous gases are present in the gap space for times after current zero up to 0.07 or 0.08 second. The illustrations and voltage breakdown curves show that subsequent breakdowns will occur through the region of brightest photographic luminescence if such still exists in the gap space. Under these conditions breakdown is started by initial stream- ers from each electrode which, however, propagate at much slower velocities than for breakdown of air under normal conditions. Time intervals as high as 30 or 40 microseconds are required for a low-impedance path to be established.

This research program is being extended to other insulating media and fault conditions and fundamental studies are being made of the dielectric-recovery mechanism.

$\mathbf{K}$ NOWLEDGE of the dielectric-recovery characteristics of powersystem insulation following flashover or breakdown is of fundamental importance in determining and improving powersystem performance. However, relatively little fundamental data have been obtained because of the complexity of the phenomena and the difficulty of performing significant tests on representative insulation media. The bulk of the data that have been gathered apply only to the circuit breaker arc or to small gaps where electrode effects dominate.

Studies of the primary insulations are important in determining probabilities of insulation failure resulting in sustained faults, permissible reclosing times for circuit breakers, and the performance of disconnect switches. . A study of the performance of simultaneous arcs through similar and dissimilar insulating media and at various distances apart on systems is required for better co-ordination of such protective devices as deion protector tubes. Dielectric-recovery studies are also of great importance in determining the characteristics of system transients during such faults as arcing grounds. The conditions under which arc extinction and reignition occur determine the characteristics and magnitudes of the resulting system transient voltages. Of particular importance in this connection are the conditions under which current zeros can be forced in arc extinction with the resulting high voltages.

Recognizing this to be a general field of considerable importance at the present time, a co-operative research program on dielectric recovery has been instigated at the High Voltage Laboratory of the California Institute of Technology. This program is being sponsored by the Department of Water and Power of the City of Los Angeles, the Kelman Electric and Manufacturing Company, and the Southern California Edison Company. The first phase of this program, discussed in this paper, deals with air insulation at normal atmospheric conditions. This medium was chosen first since it is the most fundamental one of the practical insulations and is subject to the least number of controlling factors. A limited amount of data had been obtained elsewhere on large air gaps ${ }^{1,2}$ by artificially producing faults and determining system conditions under which reignition did or did not occur. Also safe circuit-reclosing times have been determined from staged tests under a limited range of conditions. ${ }^{3,4}$ Such techniques, however, permit a very limited variation in the important parameters and require so long that either insufficient data are obtained or important variations have occurred in the conditions during the period of the test. Also many such staged tests are performed by closing in on an artificially produced or fused fault. Accurate timing of the start of the fault current thus is not obtained, and vaporized metal may be produced in the gap by the fused element.

A testing technique has been developed

Paper 50-96, recommended by the AIEE Trans mission and Distribution Committee and approved by the AIEE Technical Program Committee for presentation at the AIEE Winter General Meeting New York, N. Y., January 30-February 3, 1950 Manuscript submitted November 2, 1949; made available for printing December 2, 1949.

G. D. MCCann, J. E. Conner, and H. M. ELLIS are all of the California Institute of Technology, Pasadena, Calif.

The authors wish to acknowledge the valuable assistance rendered by $B$. N. Locanthi in the development of the electronic equipment, Sol Matt and L. H. Tejada in helping to obtain the test data, and A. T. Puder who helped with equipment construction. 


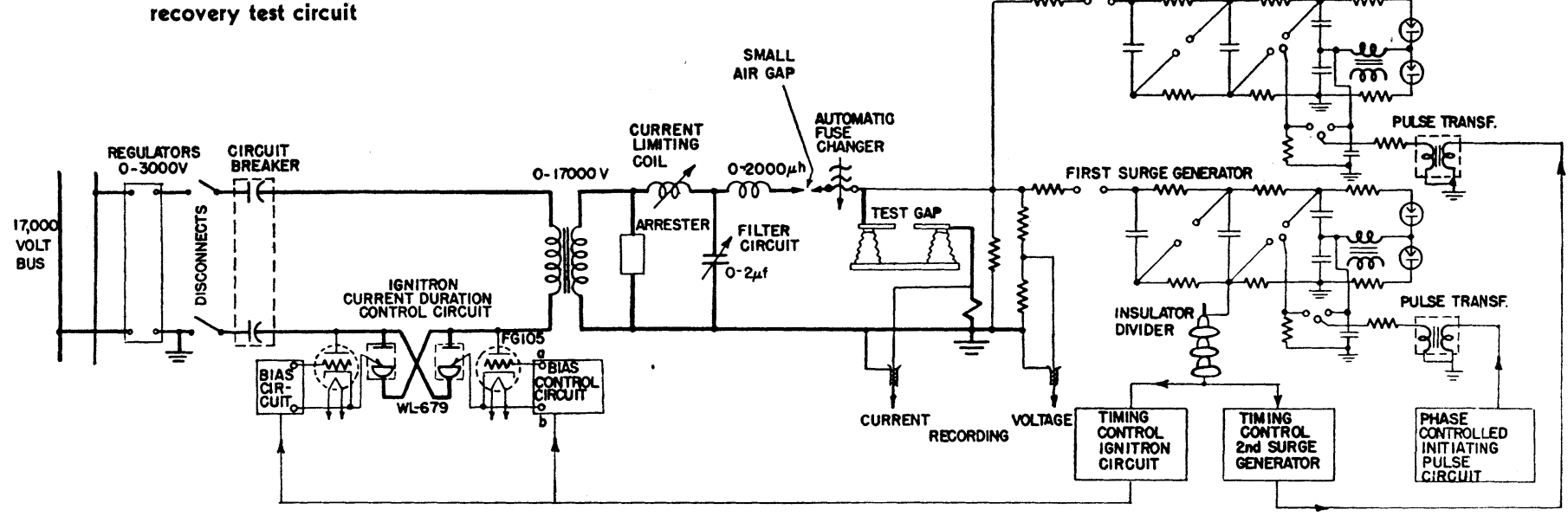

which overcomes these difficulties and is an extension of work performed earlier on lightning current faults. ${ }^{5}$ The insulation medium is first broken down by a surge generator, the fault current allowed to flow a specified time and then shut off, after which the dielectric strength of the insulating medium is tested over a wide range of time delays with a second surge generator. By this means data for complete dielectric-recovery curves can be obtained for a wide range of fault-current magnitudes and durations. Because of the very flat breakdown characteristics of air insulation, particularly while still under the influence of a predischarge, such data are applicable to all practical types of system overvoltages. This technique permits the accumulation of a large mass of data so that effects of the various important parameters can be studied and quantitative correlations made with theoretical approaches to the problem.

\section{Test Circuits and Techniques}

The main test and auxiliary control circuits are of interest not only because they represent a new technique for studying dielectric-recovery phenomena, but also because of certain new control methods that are being applied to surgegenerator testing. A general schematic diagram of the test circuit is shown in Figure 1. Figure 2 shows the main power circuit. This part of the test circuit is shown by heavy lines in Figure 1. Sixtycycle power is supplied from a $17-\mathrm{kv}$ bus through a single-phase induction regulator. This energizes a bank of four $150-\mathrm{kva} 8.6$ - to $2.4-\mathrm{kv}$ transformers which supply the actual test circuit. By various combinations of series or parallel connections a range of power currents and voltages can be supplied to the test gap. A further fine control of the fault current is obtained with the current-limiting in- ductor which can be varied in approximately one-ohm steps up to $60 \mathrm{ohms}$. Fault currents up to approximately 3,000 amperes can be obtained. The ignitron circuit shown in Figure 1 is used to control the fault-current duration if the seifextinguishing characteristics of the circuit are not to be studied directly.

To start a fault condition, the test gap is first broken down by the surge generator designated as Number 1. Its firing time is accurately synchronized with the main 60 -cycle power source by means of the phase-shifting selsyn peaking-transformer trip circuit shown in Figure 3. Thus fractions or multiples of half-cycles of fault current can be accurately duplicated. As shown in Figure 3, radar pulse transformers, rated 8 to $30 \mathrm{kv}$, have been used with excellent results for tripping the surge generators.

The facilities of the California Institute of Technology High-Voltage Laboratory include two $100-\mathrm{kv}$ surge-generator charging circuits and two separate 10 -bank, $1,000-\mathrm{kv}$ surge generators that can be used as two separate generators or as one $2,000,000$-volt generator. In this test the two separate units comprised generators Numbers 1 and 2.

When the main 60 -cycle power circuit of Figure 1 is energized, the test-gap shunt resistors are isolated by means of a small air gap to reduce their required power rating. For breaking down the test gap, surge generator Number 1 is adjusted to cause gap flashover on the wave front and thus produce a very short duration voltage pulse which (due to its high effective frequency) can be kept out of the main power circuit by means of the filter circuit shown in Figure 1. The inductor and capacitors of this circuit are shown in Figure 2 together with the test-gap and power transformers. At high frequencies this circuit is a high impedance as viewed from the test gap and thus permits suffi- cient voltage from surge generator Number 1 for gap breakdown in short times, but allows very little voltage to reach the transformer terminals.

With the use of only the one surge generator and by blocking cut-off of the ignitron circuit, it is possible to study directly the reignition characteristics of the test gap over a limited range of system recovery voltages and frequencies, as indicated by the range of variation of voltage and filter-circuit constants shown in Figure 1. Natural system frequencies from 300 to 7,000 cycles per second can be obtained.

The Number 2 surge generator is used for the second type of dielectric-recovery study in which the insulation strength of the test medium is tested at various time intervals following the end of a controlled fault current. The Number 2 surge generator and ignitron circuit are both timed by a pulse taken from the Number 1 generator as shown schematically in Figure 1. The ignitron bias control and timingcontrol circuits are shown in Figure 4. The pulse from the Number 1 generator is applied to a single-shot multivibrator circuit whose time delay in sending out a pulse can be controlled by the variable capacitor shown. This pulse is applied to the grid of a 2050 thyratron whose plate circuit supplies current to high-speed relays in the bias-control circuits of the F6105 ignitron control thyratrons, see Figure 4 . These relays have a delay time of only five milliseconds so that the ignitron circuit can cut off the fault current at any desired current zero. For fractions of a half-cycle it was found readily possible to trip the $F 6105$ grid-bias circuit by an induced pulse from the Number 1 generator. Thus the ignitrons are immediately conditioned to block off at the first current zero. The duration of fault current then is varied by the initial trip time of the Number 1 generator. 


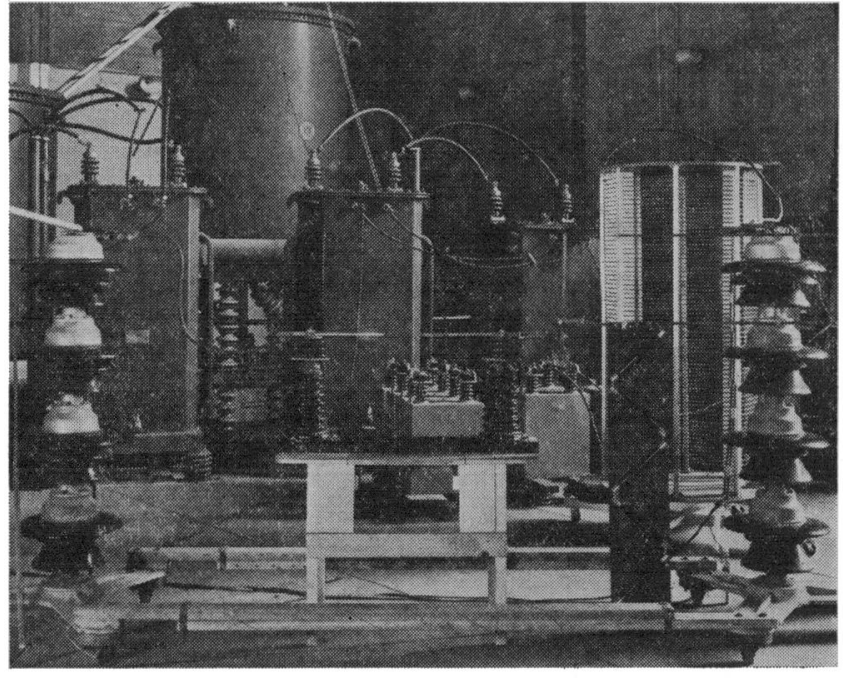

Figure 2. View of main power circuit showing test gap in foreground and power transformers in background. Capacitors and inductor of filter circuit are on right side

\section{Ten-Stage Scale-of-Two Counting Circuit}

It is desired, of course, to control accurately the time of firing of the Number 2 surge generator to study the dielectricrecovery properties of the test medium over a wide range of times after the end of the fault current. For this purpose a binary or scale-of-two flip-flop circuit ${ }^{6}$ was developed which counts pulses from a variable frequency square-wave generator. This circuit is shown in Figure 5 with its schematic diagram in Figure 6 .

Each stage of the counting circuit consists of two tubes which have two stable states and can be changed from one to the other by the appropriate trigger pulse, the two stable states being distinguished by which tube is conducting. The terminal Out of one scale-of-two is connected

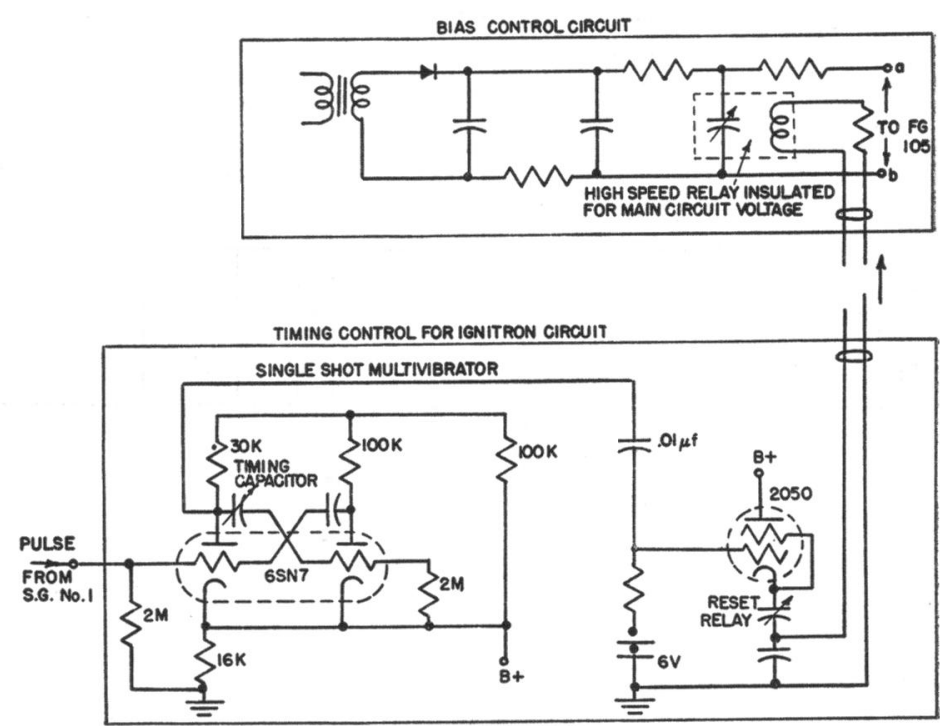

Figure 4. Timing and bias-control circuits for main ignitron fault-duration control circuit directly to the terminal In of the next scale-of-two and so on for 10 stages. When $T-2$, Figure 6 , of the preceding stage becomes conducting, a negative signal is applied to the plate of the Off tube and through the resistance-capacitance cross coupling to the grid of the On tube, which causes the flip-flop to pass to its other stable state. When $T-2$ of the preceding state becomes nonconducting the resulting positive pulse has no effect on the following scale-of-two. Each scale-of-two produces one negative pulse for each two that are applied to it from the preceding stage, so that for 10 stages a total of $2^{10}$ or 1,024 pulses input to the first stage is required to produce a complete operation of the counting circuit. Thus, by varying the frequency applied to the counting circuit a varying time delay can be obtained. The oscillator driving the square-wave generator will apply frequencies up to $80 \mathrm{kc}$, and thus for 10 stages time delays from over one minute and down to about 13,000 microseconds can be obtained. Less stages are used for shorter delays. Values less than 100 microseconds can be reproduced.

The counting circuit is triggered by a pulse from the firing of the first surge generator which changes the stable state of the $6 S N 7$ gate control tube, thus putting a positive bias on the $6 S J 7$ amplifier tube and allowing this tube to supply square waves to the first scale-of-two. The stable state of the gate-control tube is returned again to its cut-off state by a negative pulse from the counting circuit

Figure 3 (below). Control pulse circuits for timing surge-generator trip circuits

Figure 5 (right). View of timing control circuit for the number 2 surge generators showing oscillator, square wave generator, and binary counting or delay circuit
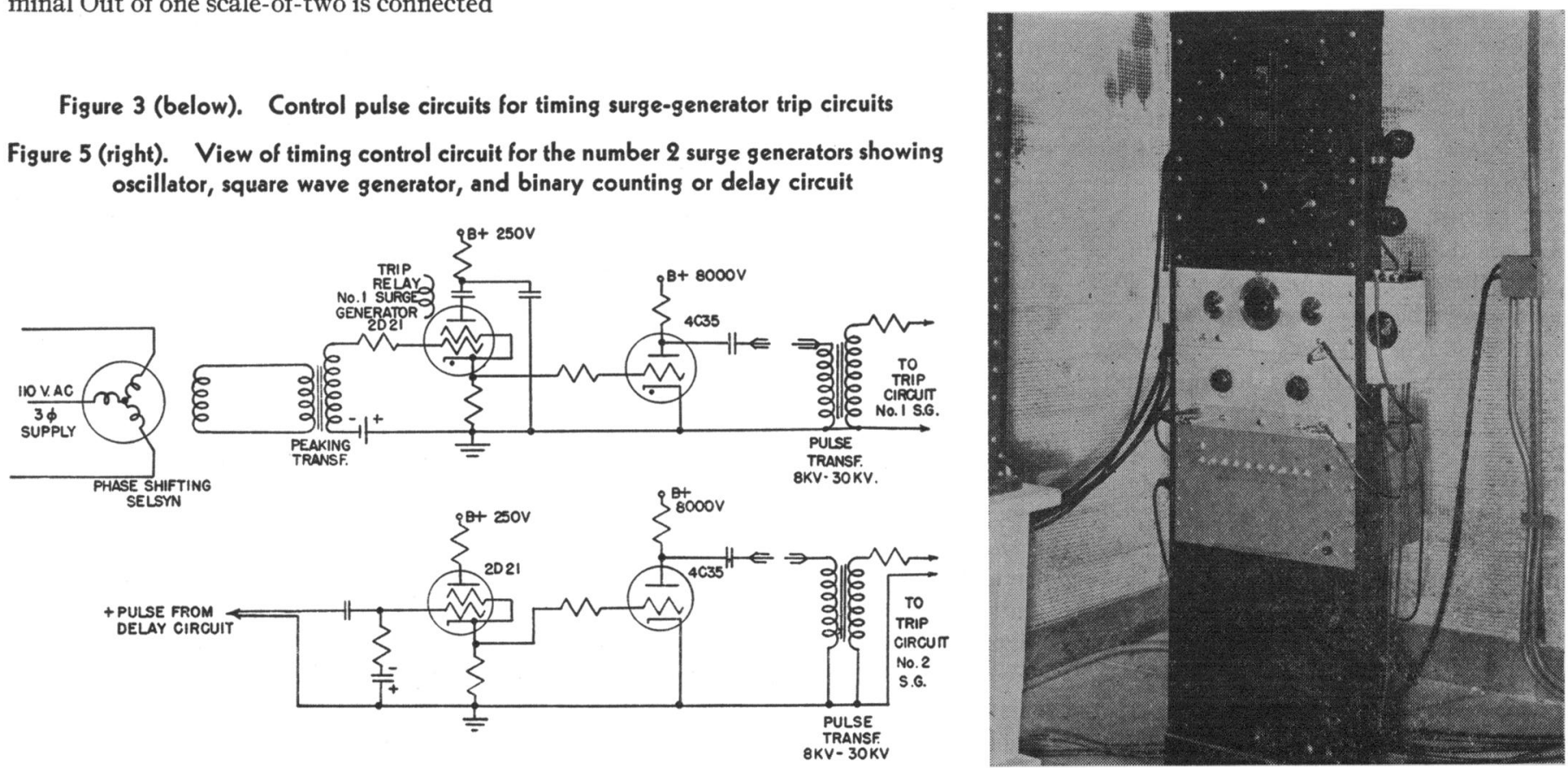


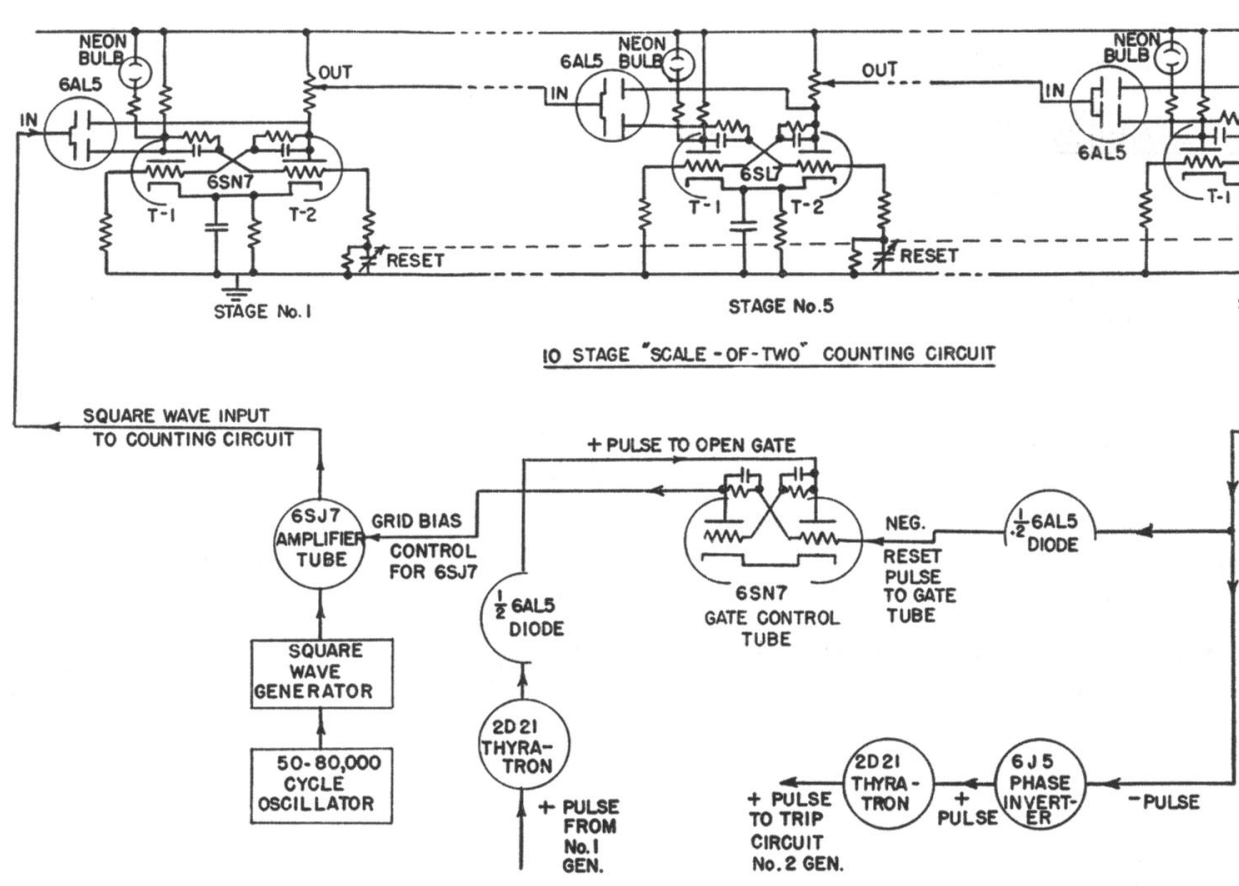

at the end of its operation which in turn negatively biases the $6 S J 7$ to cut-off and resets the counting circuit for its next operation. The control pulse out of the counter is fed to the Number 2 surgegenerator trip-pulse circuit shown in Figure 3.

\section{Automatic Fuse Changers}

Although the inductance-capacitance filter of the main power circuit, Figure 1, presents sufficient impedance for the short-duration pulse of the Number 1 generator, it is not very practical to have a filter of sufficient impedance to allow a conventional surge generator to produce a full wave with a tail of 40 microseconds or more. It was found quite easy, however, to use a series-fused gap so co-ordinated as to allow the fault current to flow but to insulate against the discharge of the Number 2 generator. An automatic fuse changer was developed to allow continu-

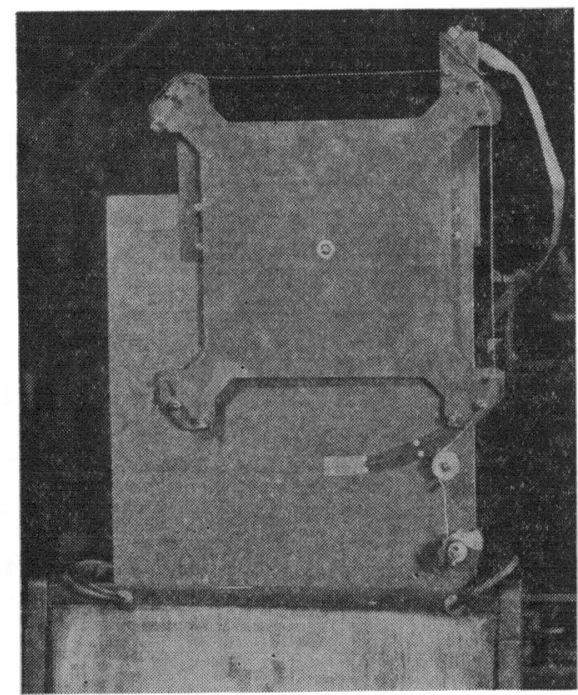

ous operation without shutting off the main power circuit between test shots. This is shown in Figure 7 . The derice pulls a small diameter copper wire from a spool around and between gap terminals at the top. The fuse holder is driven through an insulated shaft by a limitswitch position-controlled motor that accurately positions each quarter-turn of the fuse-holder rotor.

\section{RECORDING EQUIPMENT}

The complete circuit is suitable for sulation levels up to about 300 or $400 \mathrm{kv}$. As shown in Figure 1, shunts are used for Low-frequency records are obtained with a magnetic oscillograph and high-speed records with a Westinghouse cold-cathode cathode-ray oscillograph with both elecdrum-type film holder. The cathode-ray oscillograph can be tripped from the Number 1 or Number 2 generator as detesting insulating media with initial inmeasuring the gap current and voltage. trostatic sweep control and a high-speed

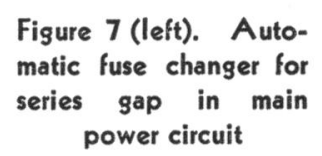

Figure 8 (right). Typical fault-current recoveryvoltage oscillogram for tests using natural system-recovery voltage. (Test 167 of Table I)
Figure 6. Schematic diagram of timing control circuit for number 2 generator sired. In addition, photographic studies of the arc path can be made with two cameras; a high-speed (3,000-frame-persecond) Eastman prism shutter-type 16millimeter movie camera and a highspeed rotating camera with film mounted on a rotating $3,600 \mathrm{rpm} 12$-inch drum which rotates past a fixed lens.

\section{Tests Using Actual System- Recovery Voltage}

Standard rod gaps were used in all studies reported here. The test-circuit insulation levels are adequate for studying gap spacings up to about 15 inches. Previous data on lightning surge currents ${ }^{5}$ had been obtained at gap spacings of 6 and 11 inches. Most of the data discussed here were obtained at the same spacings to correlate with those tests. In addition 3 -inch gaps were studied.

When using the actual system-recovery voltage of the circuit supplying the fault current, the logical procedure is to vary one of several possible parameters until critical reignition is reached. A point on a dielectric-recovery curve then can be obtained by the crest magnitude and time to crest of the system-recovery voltage when the arc just fails to restrike. The parameters which can be varied are the time constant of the circuit to control the rate of rise of the system-recovery voltage, the applied circuit voltage to control the magnitude of the recovery voltage, the magnitude of the fault cur-

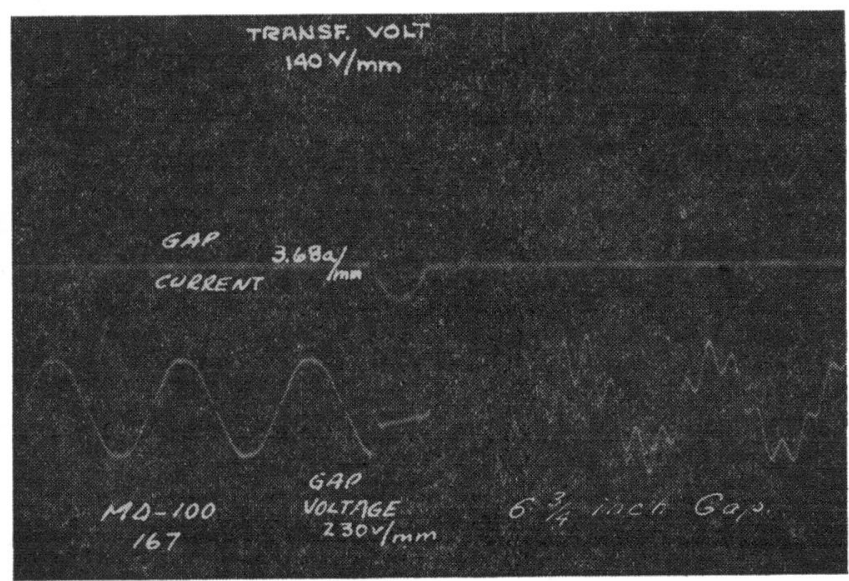



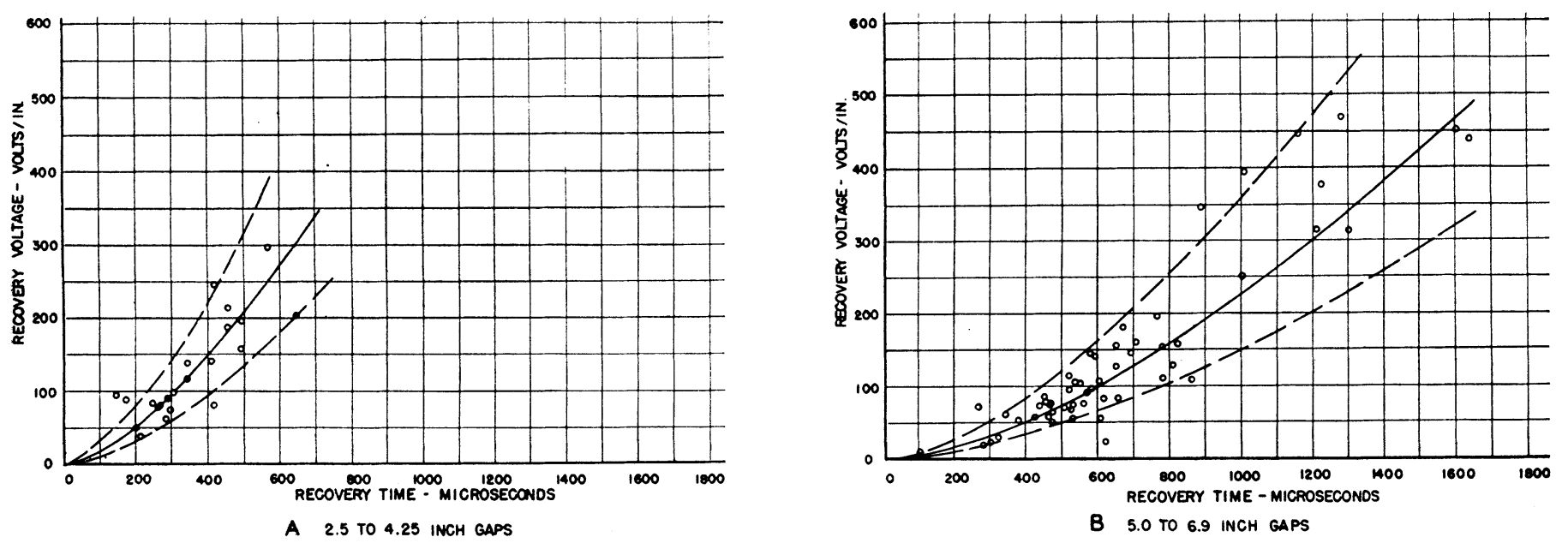

Figure 9. Data on dielectric recovery of standard rod gaps using natural system-recovery voltages. (See Table I)

A. Gap spacings from 2.5 to 4.25 inches

B. Gap spacings from 5.0 to 6.9 inches

rent, and the gap spacing. Unfortunately, it is difficult to vary any one of the above parameters without causing appreciable changes in one or more of the others. Variation of circuit voltage or system natural frequency at a fixed fault current produces a very slow test procedure since it requires an accurate readjustment of the current-limiting reactor. The best test procedure found was to set the circuit voltage, natural system frequency, and limiting reactor and then vary the gap spacing until the critical reignition condition is reached.

With gaps of the order of three to six inches, it was found that the circuit voltage had to be reduced to the order of 1,000 volts rms, or less, before arc extinction would occur naturally with fault currents of practical magnitudes. This was true with system natural frequencies as low as 300 cycles per second. Even then the currents were below 50 amperes. Therefore this phase of the study necessarily was confined to currents of this magnitude or less. Under these conditions the arc drop was appreciable and, as is to be expected, accurate reproduction of test conditions was difficult. It was found in all cases that if the arc extinguished itself naturally it did so within the first half-cycle or at the first current zero. However, for any two faults with identical settings of the controllable parameters the magnitude and duration of fault current from its start to the first current zero varied considerably. This erratic characteristic therefore requires so many tests to get a mean critical condition that enough points for even a portion of a dielectric-recovery curve can not be obtained in a short enough time so that the effects of variations in atmospheric conditions can be eliminated or studied.

Table I lists 76 sets of critical reignition test runs that were made. In the tabulation are given the applied voltage-crest fault current, per cent of one-half cycle of the fault current, crest magnitude, and time to crest of the system-recovery voltage. The current data apply to the actual oscillograms from which the recovery voltage point was scaled at the test shot on which the circuit just recovered. A typical oscillogram from which these data were obtained is shown in Figure 8. As shown in Table I, the crest current ranges from 7 to 52 amperes and the time to current zero from 18 per cent to 100 per cent of a halfcycle. Various analyses of the data were attempted, such as segregating into groups of limited current variation, but the spread in the points as plotted on dielectric-recovery curves completely

Figure 10. Comparison of time-lag or volt-time curves (obtained by two generator tests) for 3 inch rod gap with no predischarge and predischarge of one-half cycle 250-ampere crest

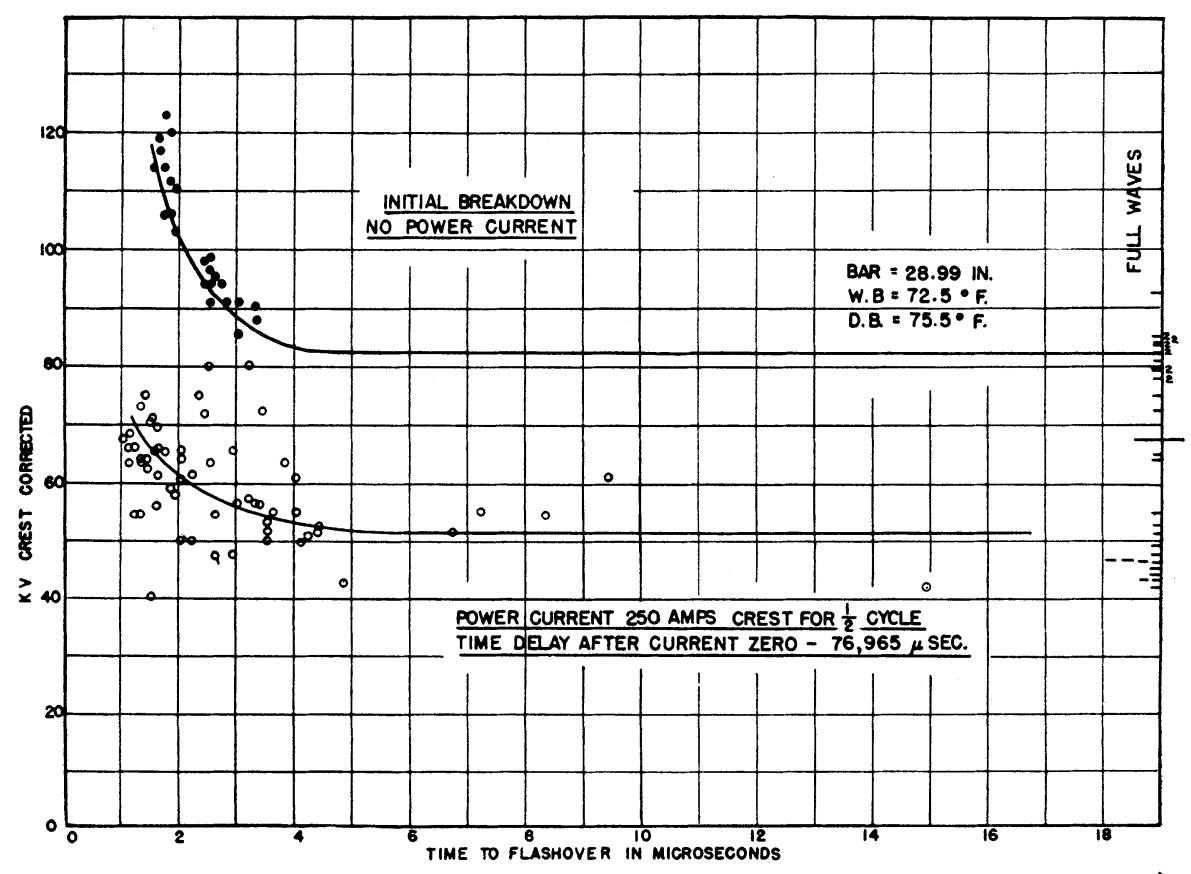


Table I. Summary of Actual System Recovery Tests

\begin{tabular}{|c|c|c|c|c|c|c|}
\hline $\begin{array}{l}\text { Oscillogram } \\
\text { Number }\end{array}$ & $\begin{array}{c}\text { Test } \\
\text { Gap, } \\
\text { Inches }\end{array}$ & $\begin{array}{c}\text { Gap } \\
\text { Crest, } \\
\text { Amperes }\end{array}$ & $\begin{array}{c}\text { Per Cen } \\
\text { Half- } \\
\text { Cycle }\end{array}$ & $\begin{array}{c}\text { Crest } \\
\text { Transformer, } \\
\text { Volts }\end{array}$ & $\begin{array}{l}\text { Crest } \\
\text { Gap, } \\
\text { Volts }\end{array}$ & $\begin{array}{c}\text { Recovery } \\
\text { Time, } \\
\text { Microseconds }\end{array}$ \\
\hline 75 . & .6 .0 & .18 .8 . & .50 & .1750 . & $.2700 \ldots$ & $\ldots \ldots 1160$ \\
\hline 78. & 6.0 & 7.2 . & .50 & $\ldots \ldots \ldots 1530$ & $.2100 \ldots$ & $\ldots 895$ \\
\hline 80. & .6 .5 & 7.7. & .50 & $\ldots \ldots \ldots 1585$ & .1660. & .1010 \\
\hline 85. & .6 .5 & 8.4 & .50 & … & $.2475 \ldots$ & $\ldots \ldots 1230$ \\
\hline 87. & .6 .5 & .11 .3 & .72 & 1560 . & $.2060 \ldots$ & $\ldots 1305$ \\
\hline $116 .$. & .6 .5 & 21.7 . & .90 & 1565 & $.3140 \ldots$ & $\ldots 1285$ \\
\hline 119. & 6.75 & .24 .0 & .86 & $\ldots \ldots \ldots 1580$ & $.2920 \ldots$ & .1640 \\
\hline 138. & $6.75 \ldots$ & .11 .4 . & .27 & $\ldots \ldots 6608$ & . 426. & 343 \\
\hline 139. & 4.0 . & 10.4 . & .36 & 609. & 372. & $\ldots 286$ \\
\hline 141. & $\ldots 3.0$ & 23.0 & .59 & 630. & 255 . & 248 \\
\hline 142. & .2 .5 & . 19.6 . & .59 & 637. & 240 . & 145 \\
\hline 149. & .3 .5 & . 12.6 . & .32 & ...661. & 195 & ... 200 \\
\hline 150. & .3 .5 & 21.8 & .59 & 627 & . 487 & ... 343 \\
\hline $151 .$. & .3 .0 & .24 .1 & .63 & 640 . & 568 & $\ldots \ldots 457$ \\
\hline 153. & 2.75 & 38.7 & .71 & 640. & 675. & $\ldots \ldots 420$ \\
\hline 154 & .2 .63$. & .27 .6 . & .64 & 625 & 375. & $\ldots 410$ \\
\hline 155. & $.2 .56 \ldots$ & 41.4 & .73 & 625 . & 765 & $\ldots 571$ \\
\hline 167. & $.6 .75 \ldots$ & .21 .8 & .86 & $\ldots \ldots 1740$ & $.3080 \ldots$ & $\ldots 1605$ \\
\hline 176. & 6.75. & .13 .4 & .77 & $\ldots \ldots 1600$ & $.2680 \ldots$ & $\ldots 1016$ \\
\hline 184 & $6.75 \ldots$ & .15 .1 . & .86 & . . . 1310. & $.2145 \ldots$ & $\ldots 1218$ \\
\hline 187. & .6 .75$. & 22.1 & .55 & $\ldots \ldots \ldots 1310$ & .1237. & $\ldots 670$ \\
\hline 193. & .6 .75$. & .25 .0 & .82 & $\ldots \ldots .906$ & .1065 & $\ldots 650$ \\
\hline 195. & 6.75 & .17.3. & .68 & 896. & 740 & $\ldots 608$ \\
\hline 199. & $.6 .0 \ldots$ & .20 .7 . & .68 & 875 & $940 \ldots$ & $\ldots 781$ \\
\hline 200 & 6.0 & 19.6 & .55 & 947. & 880. & . . 694 \\
\hline 202. & .6 .0 & .27 .6 . & .77 & $\ldots \ldots 947$ & .1206. & 770 \\
\hline $241 \ldots$ & .6 .75$. & .23 .0 & .73 & $\ldots \ldots 1040$ & .1085. & 710 \\
\hline 253. & $.3 .5 \ldots$ & 20.2 & .50 & 806 . & 260 . & 257 \\
\hline 254. & .3 .5 & . 12.1 . & .18 & 806. & 279 & 262 \\
\hline 255 . & .3 .5 & $\ldots 19.4$. & .50 & 806. & 316. & $\ldots \ldots 171$ \\
\hline 257. & .3 .5 & .16 .1 & .50 & 867. & 224 & 286 \\
\hline 258. & .3 .5 & . 10.3. & .36 & 867. & 405. & 344 \\
\hline 263. & 3.75 & .11.4. & .23 & 806 . & 281 . & $\ldots \ldots 294$ \\
\hline 265. & 3.75 & .16.1. & .45 & . 947. & $300 \ldots$ & $\ldots 417$ \\
\hline $271 \ldots$ & .3 .69 & 29.9 & .64 & . 952 . & $794 \ldots$ & 455 \\
\hline 279 & . 3.94. & .17 .2 . & .36 & 940 . & $152 \ldots$ & 214 \\
\hline 283. & $.3 .94 \ldots$ &. .27 .6 & .68 & 804. & $386 \ldots$ & 305 \\
\hline 290. & $.4 .25 \ldots$ & . 34.4 . & .59 & 934. & $837 \ldots$ & 498 \\
\hline 297. & $.5 .0 \ldots$ & . 36.3 . &. .50 & 793. & $105 \ldots$ & 286 \\
\hline 298. & .5 .0 & .56 .5 &. .50 & 793. & 216 & 271 \\
\hline 299. & .5 .25 & .56 .5 & .50 & 806. & 291. & 381 \\
\hline $301 .$. & $.5 .25 \ldots$ &. .19 .4 & $\ldots 27$ & 778 : & 71. & 105 \\
\hline 302. & $.5 .25 \ldots$ & $\ldots 44.4 \ldots$ & $\ldots 45$ & . . 804. & $185 \ldots$ & $\ldots 328$ \\
\hline $306 .$. & .5 .25$. & $\ldots 48.4$ & $\ldots 50$ & . 804 . & $306 \ldots$ & $\ldots \ldots 617$ \\
\hline $307 .$. & .6 .0 . & $\ldots 52.3$. & .50 & 764. & $435 \ldots$ & 509 \\
\hline $309 \ldots$ & .6 .0 & 48.4 & .50 & 793. & $425 \ldots$ & 530 \\
\hline 396. & $.2 .56 \ldots$ & . .32.2. & $. .90+$ & 700 . & $404 \ldots$ & 496 \\
\hline 438. & $.5 .75 \ldots$ & 21.8 & $.90+$ & 1032 & . 486 & 621 \\
\hline $441 .$. & .5 .88 & .27 .6 & $.90+$ & . 1032 . & 649 . & 785 \\
\hline $444 .$. & $.5 .63 \ldots$ & .22 .3$. & $.90+$ & .1025 . & 738. & 814 \\
\hline 449. & 6.88 & ..27.6. & $.90+$ & 985 . & .760. & $\ldots 864$ \\
\hline $452 \ldots$ & 6.38 . & .25 .3 & $.90+$ & . 962 . & 680. & $\ldots 553$ \\
\hline $454 .$. & .6 .0 & .20 .7 & $.90+$ & . 962 . & . $513 .$. & $\ldots 456$ \\
\hline 456. & .5 .94 & . 33.9 . & $.90+$ & 962 . & $868 \ldots$ & 585 \\
\hline $462 .$. & .5 .38 . & . .34.6. & $.90+$ & 962 . & $870 \ldots$ & 829 \\
\hline $471 \ldots$ & .4 .50 . & . 33.8 & $.90+$ & $\ldots \ldots \ldots 962$. & 924 & 644 \\
\hline $506 \ldots$ & $.5 .63 \ldots$ & .30 .2 & $.90+$ & $\ldots \ldots \ldots 1202$. & 813. & 595 \\
\hline $513 .$. & .6 .75$. & .36 .8 & $. .90+$ & .1147 & . $876 \ldots$ & $\ldots 657$ \\
\hline 527. & $.6 .75 \ldots$ & . .28.7. & $.90+$ & 1019 . & 726 . & 540 \\
\hline $528 .$. & $.6 .75 \ldots$ & .28 .7 & $.90+$ & ..1019. & 789 . & 723 \\
\hline $539 \ldots$ & $.6 .75 \ldots$ & $\ldots 27.2$ & $.90+$. & $\ldots \ldots 941$ & $671 \ldots$ & $\ldots \ldots 585$ \\
\hline $544 \ldots$ & $.6 .75 \ldots$ & .29 .9 & $.90+$ & $\ldots \ldots \ldots 941$ & & 520 \\
\hline $545 .$. & $6.75 \ldots$ & .24 .8 & $.90+$ & . 941. & 535 & 460 \\
\hline $551 \ldots$ & .6 .75 . & .25 .3$. & $.90+$ & 941 . & 638. & 572 \\
\hline $559 \ldots$ & .6 .75 & .17 .9 & $.90+$ & . 941. & 388. & 463 \\
\hline $561 \ldots$ & $6.75 \ldots$ & 23.0 & $.90+$ & $\therefore 941$. & . 513. & $\ldots 458$ \\
\hline $563 \ldots$ & .6 .75 & .23 .2 & $.90+$ & . 941. & $\ldots 583$. & 660 \\
\hline $565 .$. & $.6 .75 \ldots$ & .25 .3 & $.90+$. & . 835 . & 398. & 530 \\
\hline $566 \ldots$ & .6 .75$. & .34 .5$. & $.90+$ & . . 835 . & 518. & . . 534 \\
\hline $567 \ldots$ & $.6 .75 \ldots$ & $\ldots 18.4 \ldots$ & $.90+$ & . 835. & & 303 \\
\hline $568 \ldots$ & .6 .75 & .39 .1 & $.90+$ & .. 835 & 513. & $\ldots 440$ \\
\hline $571 .$. & .6 .75 & .30 .3 & $.90+$ & $\ldots 835$ & 403. & $\ldots 430$ \\
\hline 572 . & & 36.8 & $.90+$ & 835. & 525. & 473 \\
\hline $573 .$. & 6.75 . & .32 .2 . & $.90+$ & .. 835 . & 495. & $\ldots 469$ \\
\hline 575. & .6 .75 & .32 .2 . & & $\ldots 835$. & . . 488. & $\ldots 520$ \\
\hline 576. & ..6.75. & $\ldots 26.4$. & .90 & $\ldots \ldots \ldots \ldots 835$ & . 358. & $\ldots \ldots 475$ \\
\hline
\end{tabular}

duplicated and many test points obtained during essentially constant atmospheric conditions.

The method of testing the gap, at a given time interval following the end of the fault current, is to raise the voltage applied by the Number 2 generator until breakdowns occur. Then oscillograms are obtained over a sufficient voltage range to produce a volt-time or time-lag curve. It is not considered necessary to use a standard $1 \frac{1}{2}$ by 40 -microsecond wave. The volt-time curves under the conditions of a predischarge are much flatter than with no predischarge and unaffected by large variations in the tail of the test waves. This had been observed previously for the lightning surge studies. ${ }^{5}$ Typical volt-time curves both with and without a predischarge are shown in Figure 10. Two things of interest are illustrated by this figure. One is the more random nature of the data when the medium is recovering from a fault. The other is the fact that although the volttime curves plotted through points obtained in this way have less turn-up than for gaps without a predischarge, the breakdown can occur at much longer time lags. Further, it was found that for a region of time intervals (that increase with magnitude of the fault current) breakdown can occur at a relatively slow rate. This is illustrated by the oscillograms of Figure 11. This same phenomena had been observed when studying gaps recovering from high-current shortduration surge currents. ${ }^{5}$ There, however, it was found only for time intervals less than about 100 or 200 microseconds or when the gap is still carrying small currents. In the case of one or two halfcycles of power-frequency fault current this region extends to about 40,000 microseconds for 100-ampere faults and to about 70,000 microseconds for currents as high as 700 amperes. The percentage of such slow breakdown records decreases at the longer intervals. However, even though all breakdowns are at this slow rate there still exists a definite critical voltage below which breakdown will not occur. Because of this type of breakdown and the possibility of the media not supporting voltages below the apparent critical, it was considered desirable to use as long tail test waves as practicable. Surges with a time to half-value of about 80 microseconds were used for the data in all of the dielectric-recovery curves except the highest point on the 11-inch gap curve, Figure 14(B). As shown by Figure 11, partial breakdowns were also recorded at the shorter time delays after current zero.

\section{High-Speed Camera Studies}

Much valuable information has been obtained from the high-speed photographic studies, particularly from the records obtained with the 3,000-frameper-second movie camera. Portions of one of the more interesting of these records are shown in Figure 12 . The first group of frames shows the first 2,300 microseconds of the fault-current arc. The second group shows the luminous gases present in the arc 0.013 second after the start of the fault or 0.0043 second after the end of current flow. The third group shows the luminous gas in the arc just prior to the flashover produced by the Number 2 surge generator 0.041 second after the start of the fault or 0.0322 second after the end of the fault-current flow. In all cases photographed the luminous gases either moved out of the 


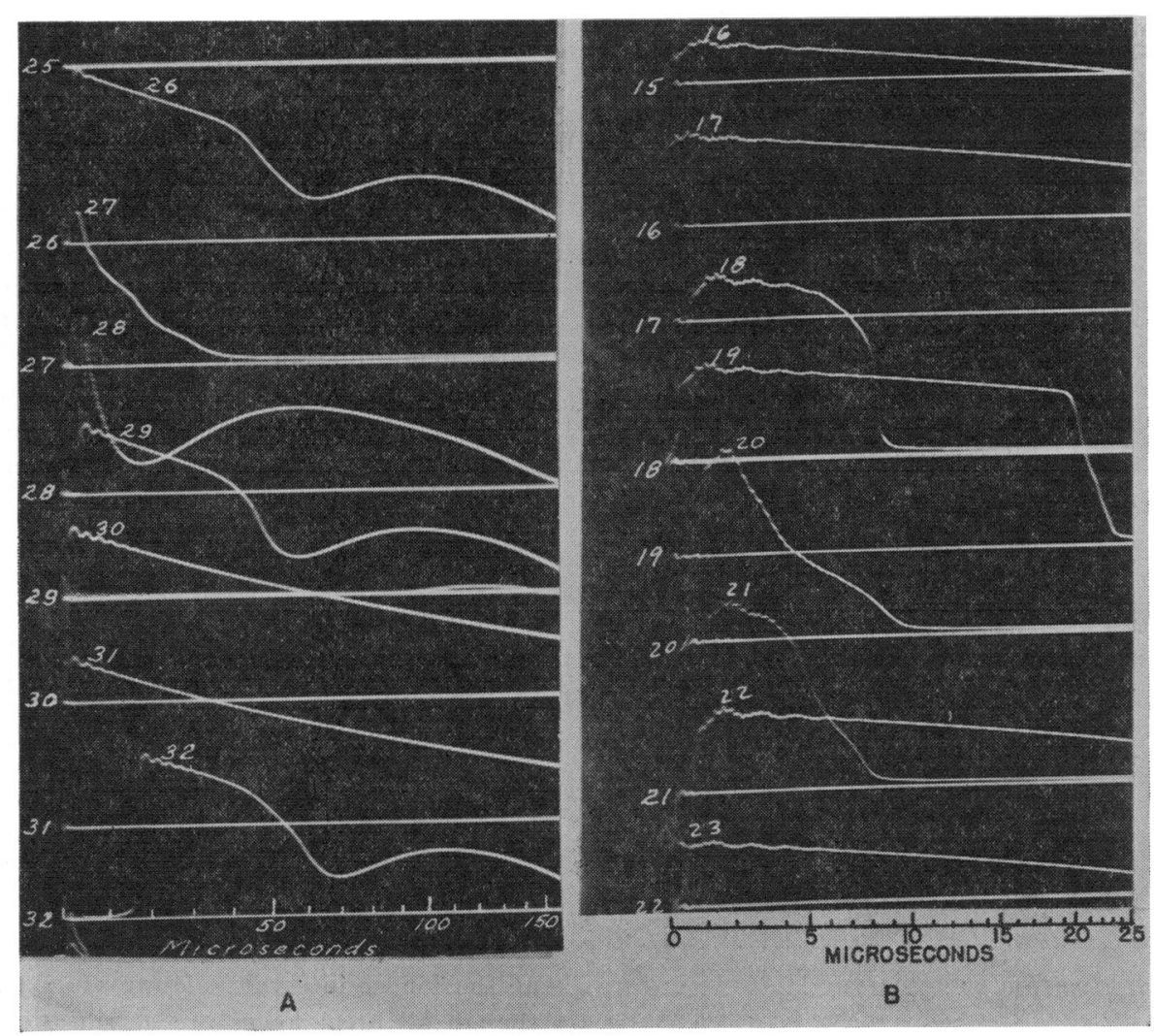

Figure 11. Typical oscillograms showing slow breakdown characteristics obtained when number 2 generator voltage is applied at short time intervals center of the gap toward the electrodes or the gases near the electrode remained luminous longer. As shown in the third group of records in Figure 12, there still remains a faint path of luminosity in the gap through which the second discharge occurred. In all recorded cases the period in which visible luminous gas remains in the central region of the gap corresponds to the maximum delay for which slow voltage breakdown is observed. The fourth group shows the second discharge, and the fifth, the luminosity which still persisted at one terminal after 0.12 second. Faint traces of luminous gas are visible in such a record as long as 0.15 to 0.17 second after the end of the fault current even though no second discharge is applied.

Detailed examination of such records shows that extensive motion in the arc path occurs even during the half-cycle of fault current. The arc develops, then expands radially (in, of course, a tortuous corkscrew path) with visible diameters of two or three inches. The luminous area shown in the frames reaches its maximum at the crest of the fault current and diminishes only slightly by the time

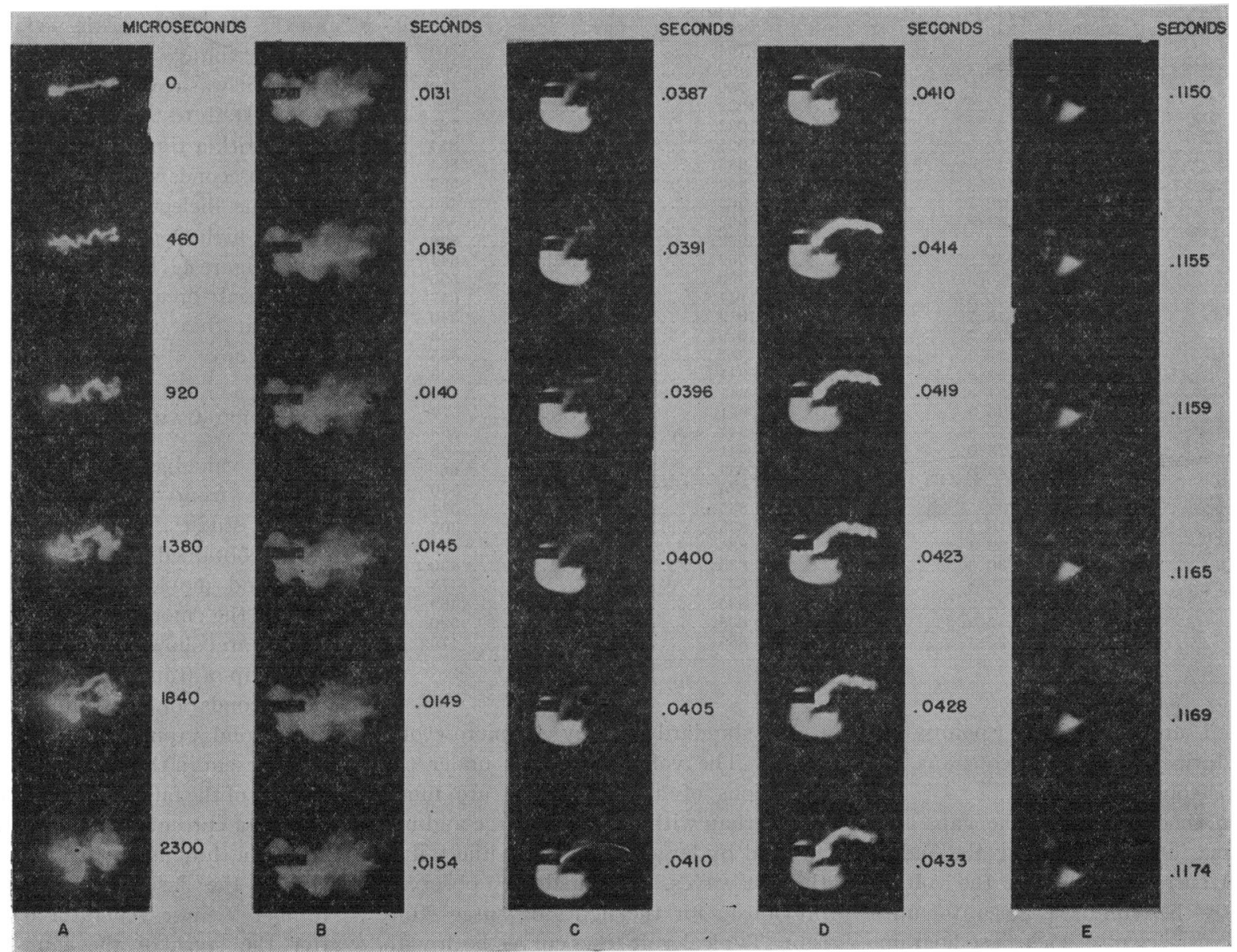

Figure 12. High-speed movie camera record of 3-inch gap discharge. Initial fault current one-half cycle of 100-ampere crest

A-Start of fault current. B-During second half-cycle of time from start of arc. C-Period just prior to second discharge. D-Period just after second discharge. E-Period toward end of visual luminescence showing ionized gas in region of one terminal only 


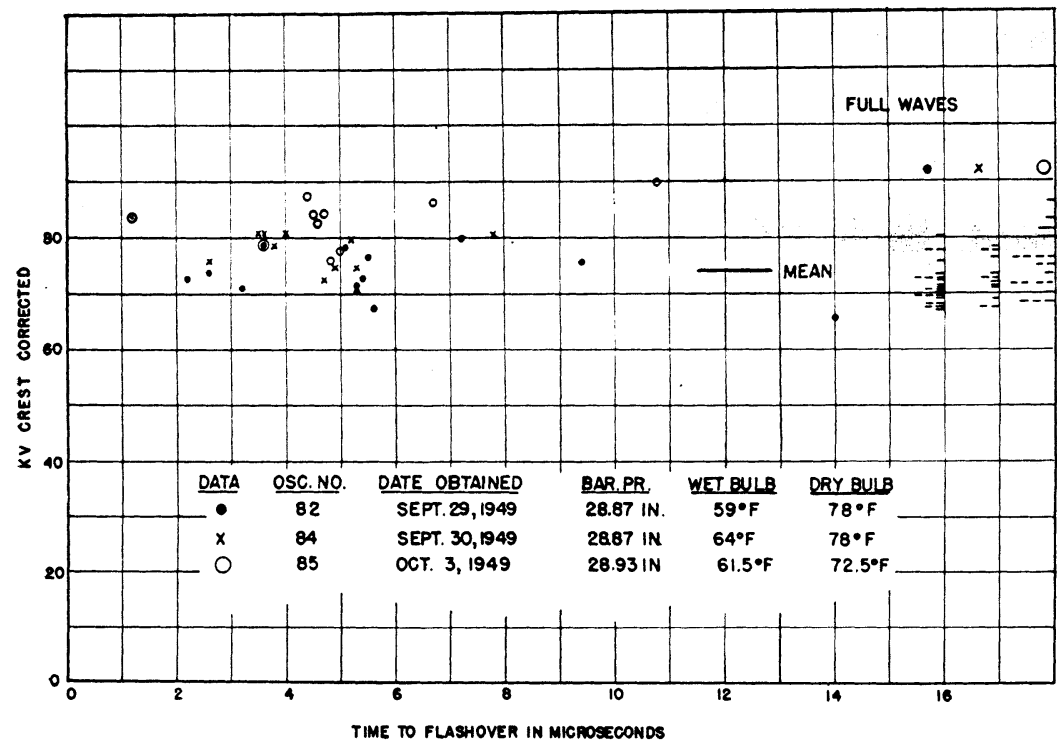

Figure 13 (above). Volt-time data for three separate runs showing effect of varying atmospheric conditions

Six-inch rod gap, $1 / 2$-cycle 10 -ampere crest fault current. Time delay after current zero 77,000 microseconds

Figure 14 (below). Dielectric-recovery curves obtained with two generator tests

A. Three-inch standard rod gaps

B. Six- and 11-inch gaps

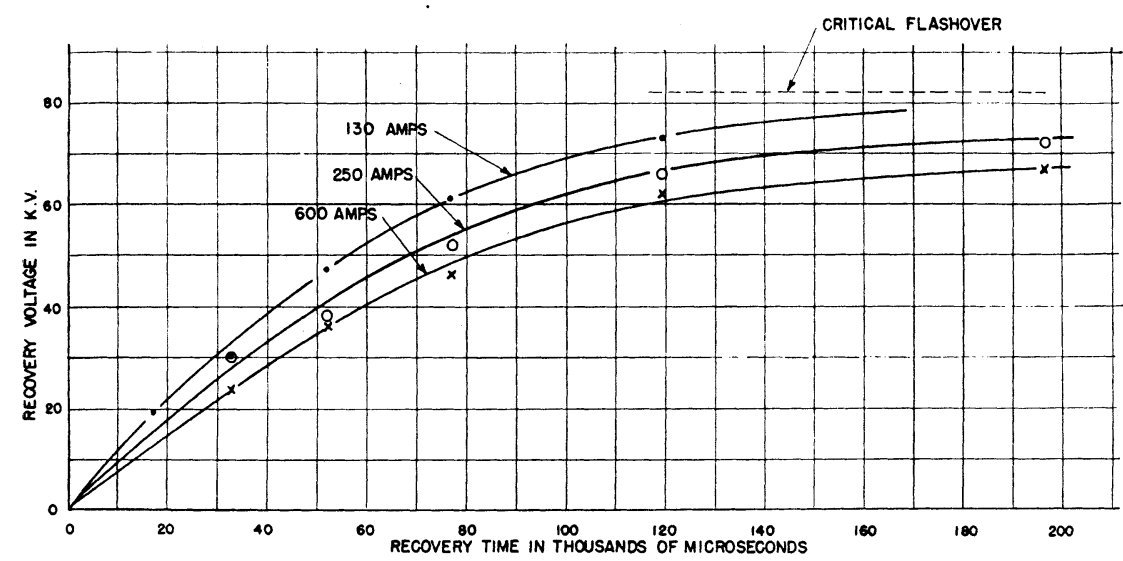

A $3 "$ ITANDARD ROD GAP - $\frac{1}{2}$ CYCLE OF FAUUT CURRENT

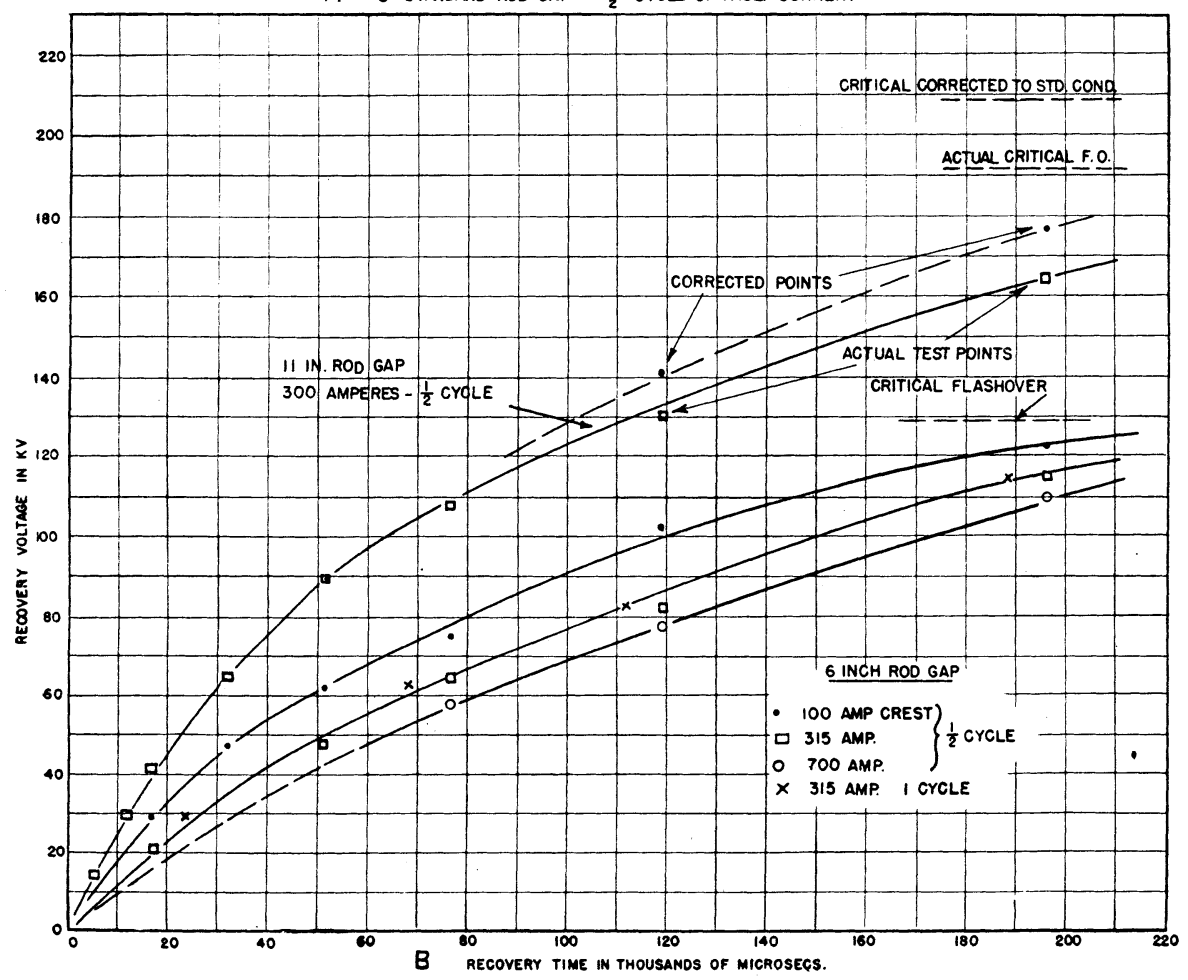

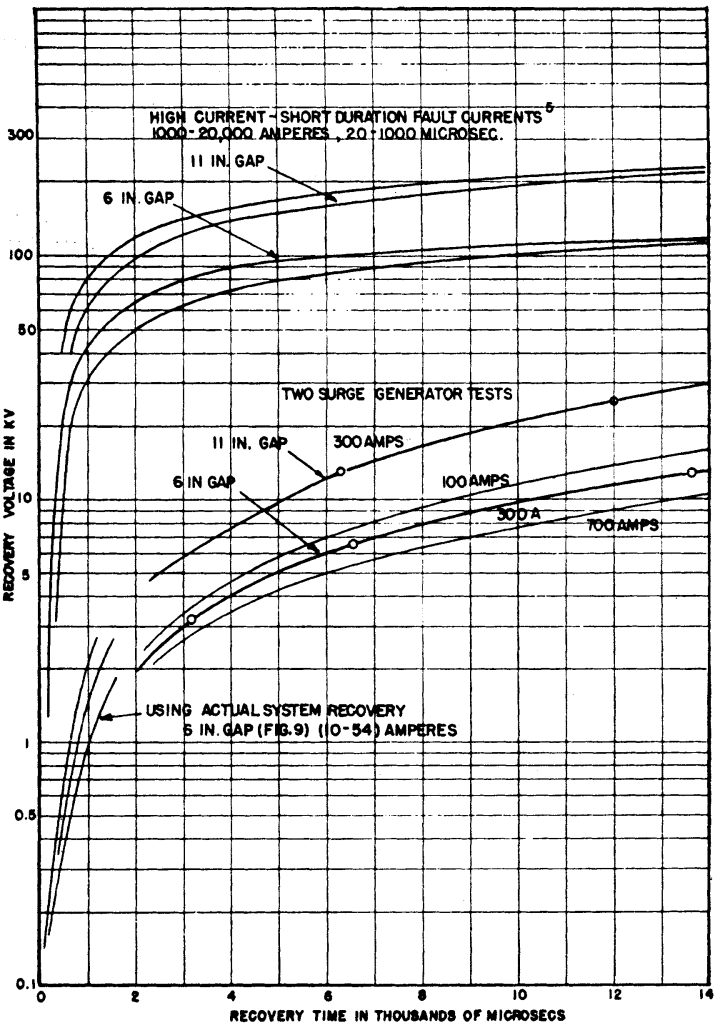

Figure 15. Comparison of dielectric-recovery characteristics of standard rod gap for different types of predischarge

the fault current reaches zero. For halfcycle faults it is quite intense for three or four more half-cycles. However, it does not drift out or away from the general region of the gap in the period that it is photographically visible.

The record in Figure 12 is of particular interest since it shows that a large time interval was required for the second discharge to build up to maximum intensity. This corresponds to the slow rate of breakdown mentioned previously. The first frame of the fourth set shows initial streamers propagating from each electrode through the trail of luminous gas. The next frame shows the intense return strcamer that completes the discharge. Of these two initial streamers the one passing through the gas of greatest luminosity is more intense itself. The time interval between these two frames is about 400 microseconds, but of course about 300 microseconds of time is lost by the shutter action and the first faint trace may have started just before the shutter closed. Thus the actual time of build-up can not be determined from such a record. Actually the longest times for breakdown to develop as recorded with the oscillograph were of the order of 30 or 40 microseconds. This was true even for very short time intervals between the end of the fault current and the application of the test voltage.

The photographic records showed that for time intervals above 70 - or 80,000 microseconds, the second discharge de- 
parts greatly from the path of visual luminosity. By this time the hot gases have disappeared in the central region of the gap and the second discharge takes a straight path between the electrodes.

\section{EfFect of Atmospheric Conditions}

The tests reported here were performed with air under natural conditions and subject to normal variations. As significant changes occurred in the weather, test runs were repeated to determine the effect. In such cases the variations between mean critical breakdown values were of the same order of magnitude as the standard correction factors to be applied to air without a predischarge. Typical data of this type is shown in Figure 13. The principal results $o^{-}$the grouping of the three runs is the correspondingly greater spread in the points. The application of the standard correction factor for varying atmospheric conditions to the test data brought the grouping closer together. In most of the data presented here, such correction factors amount to only three or four per cent at the most. Under these conditions they were applied to the data but probably have no significance. Only the two points shown in Figure $14(\mathrm{~B})$ had larger correction factors.

\section{Dielectric-Recovery Curves}

Thirty-five or forty test points appear to be adequate for establishing a critical point on a dielectric-recovery curve. However, two to four such runs were made at each time interval in an attempt to establish accurately the dielectric-recovery curves presented here. Thus each point is the result of 70 to 150 actual tests. The data obtained for longer time intervals is plotted in Figure 14 for 3-, 6-, and 11-inch horizontal rod gaps. As shown, the bulk of the data has so far been obtained for one-half cycle of power current. Shorter duration arcs have not yet been studied in detail. It has been found that the rate of recovery is relatively insensitive to longer durations. Points obtained with one or two cycles of fault current are not appreciably different than those for one-half cycle.

Comparison of the three-inch and sixinch gap data in Figure 14 shows that not only is the strength of the three-inch gap proportionately higher at any time interval but its relative rate of recovery is more rapid. Examination of critical breakdown curves for rod gaps without a predischarge shows that the relative strength of a 3 -inch gap is greater than a 6 -inch gap whereas the curve becomes a straight line above six inches. This is due to the gap configuration and probably also explains the first of the above comparisons for the cases with predischarges. The second effect is probably due to electrode cooling. The greater conductivity of the electrodes can have an appreciable effect in cooling air adjacent to each electrode. For gaps of six inches or more this effect should not be appreciable as shown by the comparison between the 11 -inch and 6-inch gap curves. These show quite similar rates of recovery and the 11-inch data have the same proportionality factor at all points. It thus appears evident that data obtained from gaps of these lengths can be extrapolated to larger gap spacings. These same considerations apply to data of Figure 9 for tests obtained using natural system recovery. Here gaps in the region of three inches have about twice the proportionate rate of recovery as 6 -inch gaps. This is somewhat higher than the proportionate ratio observed in Figure 14.

The 6- and 11-inch gap data of Figure 14 show that for fault currents of 100 to 700 amperes, time intervals of 0.02 to 0.04 second are required for the insulation to recover to one-fourth of its original strength and 0.05 to 0.08 second to recover to one-half its original strength. This region corresponds to the normal range of ratios of original transmissionline insulation strength to normal applied voltage showing the time intervals required after the interruption of the fault before it is safe to reclose the circuit breakers.

Figure 15 shows the correlation (in the region of shorter time delays) between the two types of tests described here and data previously obtained ${ }^{5}$ with high-magnitude short-duration fault currents representative of lightning surges. The very rapid recovery to lightning-surge currents is contrasted with the much slower recovery, even to power-frequency currents as low as 20 to 50 amperes. The relatively slow rates of recovery for 60-cycle fault currents make it appear unlikely that normal power-line insulation would recover after an appreciable fraction of a half-cycle of fault current flows unless after such long times that the arc has extended itself greatly by rising out of the central region of the gap. Assuming an average of 0.25 for the ratio of normal system voltage to normal insulation strength, one finds from the dielectricrecovery curves that even if the system transient or recovery voltage did not exceed normal voltage, its frequency would have to be less than 60 cycles to prevent its crossing the dielectric-recovery curve.
For a system natural frequency of 1,000 cycles per second and assuming an overvoltage of 1.5 times normal, the ratio of normal applied voltage to normal insulation level would have to be of the order of 0.01 or less. However, as shown by the dielectric-recovery curves for short-duration surges, a good probability exists for recovery at a high-frequency current zero. This was discussed in reference $\bar{j}$.

\section{FUtURE TESTS}

Unfortunately this is a very complex subject and many more tests are required before some of the important conclusions can be reached on this subject. Data have to be obtained for fault-current durations between the range of the short-duration lightning surges and appreciable fractions of one-half cycle of power frequency. The effects of appreciable air velocities and of gap positions must be studied and similar data obtained for other media such as insulator strings and wood insulation. This, together with a fundamental study of the mechanism of recovery and breakdown during the second discharge through the weakened arc path, constitute the second phase of this project now in progress.

\section{References}

1. Deionization Time of High-Voltage fault ARC PATHS, Everett J. Harrington, E. C. Starr. AIEE Transactions, volume 68, part II, 1949 , pages 997-1004.

2. Insulation flashover Deionization Times as a Factor in Applying High-Speed Reclosing Circuit Breakers, A. C. Boisseau, B. W. Wyman, W. F. Skeats. AIEE Transactions, volume 68 , part II, 1949, pages 1058-67.

3. Keeping the Line in Service by Rapid Reclosing, S. B. Griscom, J. J. Torok. Electric Journal (East Pittsburgh, Pa.), May 1933, page 201.

4. Systematic Tests with Automatic HighSpeed Reclosing in the Goesgen and Laufen burg Power System, W. Wanger. Brown Boveri Review (Baden, Switzerland), December 1945.

5. Dielectric-Recovery Characteristics of Large Air Gaps, G. D. McCann, J. J. Clark. Electrical Engineering (AIEE Transactions), January 1943 , volume 62 , pages $45-52$.

6. The Model 200 Pulse Counter, W. A Higinbothom, James Gallagher, Matthew Sands. Review of Scientific Instruments (New York, N. Y.) volume 18, number 10, October 1947, page 706 .

\section{Discussion}

O. Ackermann (Westinghouse Electric Corporation, East Pittsburgh, Pa.): Anybody who has worked on the recovery characteristics of arcs will agree fully with the authors that the subject is very complex and that it is difficult to perform significant tests, that is, tests from which any conclusions can be drawn beyond the immediate region within which they have been performed. In other words, extrapolation so far has been very risky. In order to fit isolated groups of data into a coherent pic- 


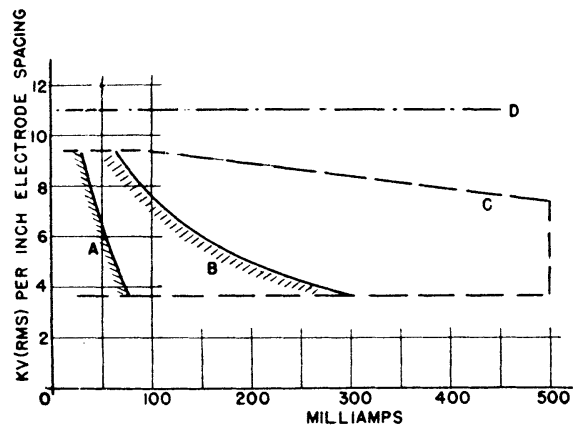

Figure 1. Interrupting ability of rod gaps (5.5 to 11 inches) at low 100-per cent powerfactor 60-cycle currents

A. Applying to both horizontal and vertical gaps: limit for clearing within four cycles B. Applying to vertical gaps only: limit for clearing at all, sometimes after as much as 30 cycles

C. Limit of explored area; horizontal gaps clear anywhere within this range, sometimes taking up to 70 cycles

D. Average gradient for initial breakdown of rod gap

ture, a reliable core or outline is needed such as now is taking shape in the hands of the authors of this paper.

A few years ago the writer found it necessary to check into the clearing ability of the air gaps employed in series with ex pulsion-type arresters for transmission lines, the question being, what leakage current flowing over the outside of the tube or over an insulator supporting the tube can be interrupted by the series gap. Tests were made on rod gaps ranging from $5^{1 / 2}$ to 11 inches, both in vertical and in horizontal position; the currents were limited to 500 milliamperes and less. The data were coordinated on the basis of kilovolts rms per inch of gap spacing; the results are shown in Figure 1 of the discussion. Horizontal and vertical gaps clear equally well in the current and voltage range where the clearing requires only a few cycles, that is, while the arc still is in the more or less direct line between the two electrodes. In the subsequent cycles some flaring out and lengthening occurs even in the vertical arc, but the process cannot go very far and the arc will not clear at all unless it can do so within the first 30 cycles. Horizontal arcs never failed to clear within the range of the performed tests, which is indicated by line $C$ in Figure 1 of the discussion.

In all these tests the current was entirely resistance limited; therefore, after current zero, the voltage reappeared across the gap in the form of a symmetrical 60-cycle sine wave, starting from zero. In the critical clearing tests indicated by line $A$ in Figure 1 of the discussion, the dielectric-recovery curve of the gap must have more or less followed that sine wave. To see how this fits into the picture presented by the authors in Figure 15 of the paper, we select the 5 -kv 66 -milliampere point of our line $A$, convert the voltage to that for an 11-inch gap, that is, $55 \mathrm{kv}$ rms, $78-\mathrm{kv}$ crest, and plot the corresponding sine wave in Figure 15. This is done in the Figure 2 of the discussion, and it appears that the new curve, although arrived at by an approximative method, fits fairly well into the general pic-

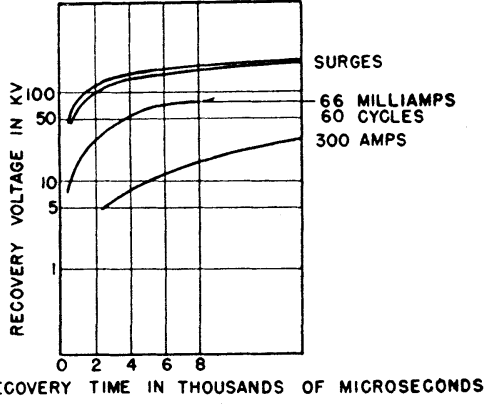

Figure 2. Comparison of dielectric-recovery characteristics of an 11-inch rod gap for different types of predischarge

ture and adds information on one of the numerous fringe areas to this extensive problem.

G. D. McCann, J. E. Conner, and H. M. Ellis: The authors were happy to receive the additional information provided by Mr. Ackermann's discussion and to see the agreement with data presented in the paper. As pointed out by Mr. Ackermann, the very great complexity of this subject makes it extremely risky to rely on a few isolated points for reliable information on dielectric recovery or try to extrapolate into unconfirmed regions.

It was for these reasons that so much effort has been devoted by the authors to the development of a reliable and sufficiently rapid dielectric-recovery testing technique that makes possible a comprehensive study of this subject. 